\title{
The Randomized Controlled Trials Rehabilitation Checklist
}

\section{Methodology of Development of a Reporting Guideline Specific to Rehabilitation}

\author{
Stefano Negrini, MD, Susan Armijo-Olivo, MScPT, PhD, Michele Patrini, MD(s), Walter R. Frontera, MD, PhD, \\ Allen W. Heinemann, PhD, Wendy Machalicek, PhD, John Whyte, MD, PhD, \\ Chiara Arienti, $M S c, D O, P h D(s)$, and RCTRACK Promoters
}

\begin{abstract}
Background: One of the goals of Cochrane Rehabilitation is to strengthen methodology relevant to evidence-based clinical practice. Toward this goal, several research activities have been performed in rehabilitation literature: a scoping review listed the methodological issues in research, a study showed the low clinical replicability of randomized controlled trials, two systematic reviews showed the relevant items in reporting guidelines, and a series of articles discussed main methodological issues as a result of the first Cochrane Rehabilitation Methodological Meeting (Paris 2018). The need to improve the quality of conduct and reporting of research studies in rehabilitation emerged as a relevant task. The aim of this article is to present the Randomized Controlled Trial Rehabilitation Checklists (RCTRACK) project to produce a specific reporting guideline in rehabilitation.
\end{abstract}

Methods: The project followed a combination of the CONsolidated Standards of Reporting Trials and EQUATOR Network methodologies. The project includes five phases. The first is kick-off, first consensus meeting and executive and advisory committee identification. The second is literature search and synthesis, where eight working groups will produce knowledge synthesis products (systematic or scoping reviews) to compile items relevant to reporting of randomized controlled trials in rehabilitation. The topics will be as follows: patient selection; blinding; treatment group; control group and cointerventions; attrition, follow-up, and protocol deviation; outcomes; statistical analysis and appropriate randomization; and research questions. The third is guidelines development, which means drafting of a document with the guidelines through a consensus meeting. The fourth is Delphi process consensus, a Delphi study involving all the rehabilitation research and methodological community. The fifth is final consensus meeting and publication.

From the Department of Biomedical, Surgical and Dental Sciences, University of Milan, Italy (SN); IRCCS Istituto Ortopedico Galeazzi, Milan, Italy (SN); University of Applied Sciences, Faculty of Business and Social Sciences, Osnabrück, Germany (SA-O); Research Centre, Faculty of Rehabilitation Medicine, University of Alberta, Edmonton, Alberta, Canada (SA-O); IRCCS Fondazione Don Carlo Gnocchi, Milan, Italy (MP, CA); Department of Physical Medicine, Rehabilitation, and Sports Medicine, University of Puerto Rico School of Medicine, San Juan, Puerto Rico (WRF); Department of Physical Medicine and Rehabilitation, Feinberg School of Medicine, Northwestern University and Shirley Ryan AbilityLab, Chicago, Illinois (AWH); Department of Special Education and Clinical Sciences, College of Education, University of Oregon, Eugene, Oregon (WM); and Moss Rehabilitation Research Institute, Albert Einstein Healthcare Network, Elkins Park, Pennsylvania (JW)

All correspondence should be addressed to: Michele Patrini, MD(s), IRCCS

Fondazione Don Carlo Gnocchi, Via Capecelatro, 66 - 20148 Milan, Italy;

Susan Armijo-Olivo, MScPT, PhD, Faculty of Business and Social Sciences,

Albrechtstraße 30 - 49076 Osnabrück, Germany.

Financial disclosure statements have been obtained, and no conflicts of interest have been reported by the authors or by any individuals in control of the content of this article.

Copyright (C) 2019 Wolters Kluwer Health, Inc. All rights reserved.

ISSN: 0894-9115

DOI: 10.1097/PHM.0000000000001370
Conclusions: The RCTRACK will be an important contribution to the rehabilitation field and will impact several groups of rehabilitation stakeholders worldwide. The main goal is to improve the quality of the evidence produced in rehabilitation research. The RCTRACK also wants to improve the recognition and understanding of rehabilitation within Cochrane and the scientific and medical community at large.

Key Words: Rehabilitation, Research, Randomized Controlled Trial, Checklist

(Am J Phys Med Rehabil 2020;99:210-215)

C linical research should inform the process of making decisions in clinical practice and randomized controlled trials (RCTs) are considered the criterion standard in the evaluation of the effectiveness of treatment. The quality of methodology, both conducting and reporting, is fundamental for replicability and the applicability of results in clinical practice. ${ }^{1}$

In the last $20 \mathrm{yrs}$, rehabilitation research publication has consistently grown $3 \%$ per year, and in $2017,19.3 \%$ and $28.2 \%$ of the total production of scientific articles for rehabilitation and physical therapy (respectively) were randomized controlled trials and systematic reviews; the corresponding rate was $11.3 \%$ for drug therapy. ${ }^{2}$ Moreover, $9.4 \%$ (1 in every 11$)$ of Cochrane Reviews are directly relevant to the practice of rehabilitation. ${ }^{3}$ Despite these encouraging findings, there are difficulties for rehabilitation in meeting the traditional methodological standards to produce high-quality evidence. These problems include the complexity of the populations studied, the wide variety of interventions, the difficulty and often inability to blind patients and clinicians in several intervention contexts, the heterogeneity of the patient outcomes, the difficulty in replicably operationalizing therapeutic interventions, and the selection of comparison groups. ${ }^{4}$ In addition, the person-centered nature of rehabilitation intervention often conflicts with the need for homogenous and standardized study protocols. ${ }^{5}$ For this reason, Cochrane Rehabilitation, which was founded in 2016 as a "field" to be a bridge between Cochrane and the rehabilitation community worldwide, ${ }^{6,7}$ started a series of activities to improve the quality of methodology in rehabilitation research (https:// rehabilitation.cochrane.org/).

In 2017, a survey of rehabilitation stakeholders on methodological issues in rehabilitation research highlighted that the most important areas of concern or interest were $(a)$ how study questions were developed, $(b)$ how the PICOs (patient, intervention, control/comparison intervention, outcome) had been interpreted and reported in past randomized controlled trials, and $(c)$ the generalizability of studies included in systematic reviews. ${ }^{8}$ 
In 2018, the first Cochrane Rehabilitation Methodology Meeting (CRMM), an intensive two-day workshop, was held before the 12th World Congress of the International Society of Physical and Rehabilitation Medicine in Paris, France, to address these concerns. A series of methodological problems in rehabilitation research were discussed and the results published in a special issue of the European Journal of Physical and Rehabilitation Medicine. ${ }^{9}$ The discussion focused on the future development of studies and tools for improving the conduct and reporting of primary studies and systematic reviews in healthrelated rehabilitation interventions. The articles of that special issue varied widely, from philosophical discussions to empirical evidence articles, but issues of complexity and clinical heterogeneity emerged as common themes across the articles. ${ }^{9-18}$

This discussion continued during the Second CRMM held in Kobe, Japan, in June 2019. The articles published in the present issue of the American Journal of Physical Medicine and Rehabilitation summarize those discussions. ${ }^{19-23}$ At the Kobe CRMM, the team presented and discussed a "catalog" of all methodological issues in rehabilitation research. ${ }^{4}$ The main issues are the following: the problem with the application of the standard RCT design; the absent definition of core outcome sets; poor description of the interventions; weak methodological (conduct) and reporting quality; limited applicability in clinical practice; lack of blinded assessor; inadequate randomization methods or inadequate allocation concealment; and inadequate description and recruitment of participants.

In preparation of the Kobe CRMM, several research activities had been performed by Cochrane Rehabilitation. In addition to the catalog of common methodological issues in rehabilitation, the Replicability in Rehabilitation clinical Practice study ${ }^{1}$ evaluated whether RCTs in rehabilitation included all details for replication of the studied intervention in different clinical settings. The results of this study demonstrate problematically low clinical replicability in rehabilitation studies particularly of items related to human factors (typical of rehabilitation), such as the interventionist skills and experience, and the relationships with the patients and into the team. That study ${ }^{1}$ also showed no differences between high- and low-quality RCTs, as it could be judged according to the CONsolidated Standards of Reporting Trials (CONSORT) checklists. The conclusion was that the ability to replicate the study treatment in a clinical setting was unrelated to the quality of the RCT, as judged by the classical reporting methodological checklists. Consequently, the development of specific guidelines for reporting (and conduct) to improve research studies in rehabilitation could be useful.

Along with these studies, Armijo-Olivo et al. ${ }^{24-27}$ published a series of meta-epidemiological studies looking at the influence of methodological biases on physical therapy intervention effects. In addition, they looked at the current checklists related to reporting and conduct relevant to physical therapy. ${ }^{28-30}$ These are the only studies evaluating the field in this perspective, even if rehabilitation is wider that physical therapy, which is mostly but not totally included. A major conclusion from this work was that there are many relevant checklists; however, there is extensive item variation across tools. Some of the items are linked to reporting and others to conduct. No agreement exists on the optimal tool (reference standard) or core set of quality criteria needed to determine the reporting quality and the risk of bias (RoB) in RCTs in the physical therapy. Most of these tools were neither developed nor validated using scientifically rigorous methods. ${ }^{28}$ In addition, using different tools to evaluate primary research included in systematic reviews can lead to discrepancies and skewed interpretations of their results, ${ }^{26,31-33}$ ultimately biasing recommendations for clinical care. A possible explanation for the variation of items in existing tools could be the fact that rehabilitation often combines biological and behavioral component ${ }^{34}$ and that these components are usually addressed in different reporting guidelines. These preliminary results call for an in-depth analysis of items that should be used to assess reporting and RoB of RCTs in the rehabilitation field. Further empirical evidence on the use of individual items and the psychometric properties of these tools are also needed.

Unified recommendations including all the items needed for rehabilitation studies production and reporting with an emphasis on functioning would be helpful to researchers and editors in the field. ${ }^{10}$ These recommendations could also serve as a tool for knowledge translation and education, providing all the needed details in an appropriate language to the rehabilitation audience. For all these reasons, we launched the Randomized Controlled Trial Rehabilitation Checklist (RCTRACK) project.

\section{Objectives}

The aim of RCTRACK is to produce a checklist of items to be reported in publication of RCTs in rehabilitation. The final RCTRACK checklists could be a stand-alone checklists or a specific add-on (not substitution) to one of the CONSORT checklists. This issue will be decided during the process. This work will also be preliminary to a twin project to develop conduct guidelines.

\section{Design}

The RCTRACK has been developed following the process used by the CONSORT Group ${ }^{35,36}$ and adapting the methodology to the EQUATOR Network suggestions (http://www.equatornetwork.org/toolkits/developing-a-reporting-guideline/). The project has been deposited in the EQUATOR Network repository (http://www.equator-network.org/library/reporting-guidelinesunder-development/reporting-guidelines-under-developmentfor-clinical-trials/\#RCTRACK). An overall view of the project is reported in Table 1.

RCTRACK includes the following five phases:

1) Kick-off, including the first consensus meeting; the executive and advisory committees identification; the registration of the title and first project synthesis in the Equator Network repository; and the final definition and publication of the project. This phase concludes with this article;

2) Literature search and synthesis: it will include the studies of the RCTRACK Working Groups (RWGs) in preparation for the second consensus meeting (Table 2);

3) Guidelines development: it will compile information from the previous phases and generating a draft document with the guidelines through a consensus meeting;

4) Delphi process consensus: it will include a Delphi study involving all the rehabilitation research and methodological community; 
TABLE 1. Overview of the Randomized Controlled Trials in Rehabilitation Checklist project

Preliminary works Scoping review on methodological issues in research ${ }^{4}$

Study on replicability of RCTs ${ }^{1}$

Two systematic reviews on items relevant to physical therapy in reporting guidelines ${ }^{27,28}$

First Cochrane Rehabilitation Methodology Meeting (Paris 2018) about main methodological issues in rehabilitation ${ }^{9}$

Current project

Methods

RCTRACK project

June 8, 2019

Kick-off

Consensus Meeting during the second Cochrane Rehabilitation Methodology Meeting in Kobe (Japan)

June 2019-March 2020

Literature search and synthesis

Executive and Advisory Committees identification

Working Groups for knowledge synthesis products (systematic or scoping reviews) on:

1. Patient selection

2. Blinding

3. Treatment group

4. Control group and co-interventions

5. Attrition, follow-up, and protocol deviation

6. Outcomes

7. Statistical analysis and appropriate randomization

8. Research questions

March 3-4, 2020

Guidelines development

Consensus Meeting during the fourth Cochrane Rehabilitation Methodology

Meeting in Orlando (USA)

Drafting of a document with the guidelines through a consensus meeting

March 2020-December 2020

Delphi study involving all the rehabilitation research and methodological community

Delphi process consensus

January-June 2021

Consensus Meeting (to be defined)

Final Consensus and publication

Paper drafting, internal review, and submission

5) Final consensus meeting and publication: it will compile the results from the Delphi study and will generate a final document with the recommendations. In addition, this phase will lead to the final publication.

\section{Funding}

This project is supported by Cochrane Rehabilitation, providing administrative assistance and coordination of the activities (ie, secretarial support) through its own funding; however, individual participants are self-funded. They volunteer their time, travel, and accommodation expenses when required. The consensus meetings during 2nd and 4th CRMM, in Kobe and Orlando, respectively, are supported by the International Society of Physical and Rehabilitation Medicine.

\section{METHODS}

The project is chaired by the Director of Cochrane Rehabilitation (SN) and managed by the Headquarters of Cochrane
Rehabilitation (CA, MP). It is led by an executive committee and supported by an advisory committee.

\section{Phase 1: Kick-off}

The Kick-off Meeting was held during the 2nd CRMM in Kobe, Japan, on June 8, 2019. Participants included the promoters of the RCTRACK. During the meeting, the following topics were presented, discussed, and approved:

1. The preliminary studies on the methodological issues in rehabilitation research described above in the introduction section;

2. The methodology of RCTRACK as reported in this article;

3. The number, leaderships, methodology, and topics of the RWGs;

4. The composition and roles of the committees (Executive and Advisory).

The Chair of RCTRACK received a mandate to contact the leaders of the RWGs and the members of the committees and to define their participation.

TABLE 2. The Randomized Controlled Trials in Rehabilitation Checklist Working Group and respective leaders

\begin{tabular}{lcc}
\hline & RCTRACK Working Group & Leader(s) \\
\hline 1 & Patient selection (population) & Thorsten Meyer (Ger) \\
2 & Blinding & Allen Heineman (USA) \\
3 & Treatment group & Wohn Whyte (USA) \\
4 & Control group and co-interventions & Levack (Nzl) \\
5 & Attrition, follow-up, and protocol deviation & Susan Armijo-Olivo (Ger/Can), Wendy Machalicek (USA) \\
6 & Outcomes & Pierre Coté (Can) \\
7 & Statistical analysis and appropriate randomization & Chimbare (Can) \\
8 & Generalities on research (design, question, effectiveness) & Chiara (Ita) \\
\hline
\end{tabular}




\section{Executive Committee}

The duties of the Executive Committee are to evaluate and approve the project, define the first draft of the RCTRACK guidelines, recruit participants for the Delphi Consensus, and approve the final guidelines.

The Executive Committee members includes $(a)$ rehabilitation professionals with clinical and methodological expertise (who have published RCTs or articles on methodological issues in rehabilitation research), (b) clinical epidemiologists who have published articles on methodological and statistical issues in nonpharmacological treatments, (c) Chief Editors of rehabilitation journals, and $(d)$ representatives of groups dealing with evidence and methodology in rehabilitation and of Cochrane methods groups. The leaders of the RWGs have also been included in the Executive Committee as ex officio members. As suggested by previous experiences in the development of guidelines, ${ }^{35,36}$ we aimed to limit the number of participants to a maximum of 30 , to control costs and maximize interaction during the meetings.

The Executive Committee includes: Chiara Arienti (Ita), Susan Armijo-Olivo (Ger/Can), Leighton Chan (USA), Pierre Côté (Can), Anne Cusick (Aus), Raju Dhakal (Npl), Julia Patrick Engkasan (Mys), Giorgio Ferriero (Ita), Walter Frontera Roura (USA), Francesca Gimigliano (Ita), Andrew J. Haig (USA), Allen W. Heinemann (USA), Thomas Hoogeboom (Nld), Alan Jette (USA), Carlotte Kiekens (Bel), Friedbert Kohler (Aus), Dinesh Kumbhare (Can), William Levack (Nzl), Wendy Machalicek (USA), Antti Malmivaara (Fin), Thorsten Meyer (Ger), Paul Montgomery (Gbr), Stefano Negrini (Ita), Randolph Nudo (USA), Aydan Oral (Tur), Dominic Pérennou (Fra), Susan Slade (Aus), Gerold Stucki (Che), and John Whyte (USA).

\section{Advisory Committee}

The Advisory Committee has the function of providing methodological support throughout the project, recruiting participants for the Delphi Consensus process, and supporting the final application of the guideline.

The Advisory Committee includes members invited but not able to commit to the Executive Committee, who nevertheless were eager to support the RCTRACK project. The Advisory Board of Cochrane Rehabilitation members were included in the RCTRACK Advisory Committee as ex officio participants.

The Advisory Committee includes: Masami Akai (Jpn), Liliana Alvarez (Can), Clare Ardern (Swe), Marcas M. Bamman (USA), Carsten Bogh Juhl (Dnk), Kristian Borg (Swe), Michael Brown (USA), Nicholas Christodoulou (Cyp), Alarcos Cieza (Che), Roberto D’Amico (Ita), Christopher Eccleston (Gbr), Franco Franchignoni (Ita), Rolf Frischknecht (Che), Frane Grubisic (Hrv), Christoph Gutenbrunner (Ger), Tracey Howe (Gbr), Elena Ilieva (Bgr), Gert Kwakkel (Ned), Sallie Lamb (Gbr), Jianan Li (Chn), Leonard S.W. Li (Hkg), Patricia Logullo (Gbr), Luz Helena Lugo (Col), Jan A. Monsbakken (Nor), Silvia Minozzi (Ita), Ann Moore (Gbr), Alex Pollock (Gbr), Farooq Rathore (Pak), Holger Schünemann (Can), Beverly Shea (Can), Henk Stam (Ned), Luigi Tesio (Ita), Peter Tugwell (Can), Derick Wade (Gbr), Linda J. Woodhouse (Aus), Sam Wu (USA), Abena Yeboaa Tannor (Gha), and Mauro Zampolini (Ita).

\section{Phase 2: Literature Search and Synthesis}

During the kick-off meeting at the 2nd CRMM in Kobe, Japan, 2018, the scoping review of the methodological issues in rehabilitation research ${ }^{4}$ and the systematic reviews on the existing tools used to guide the conduct and RoB assessments in the area of physical therapy ${ }^{27}$ were presented and discussed. These studies provided a categorized list of the issues in rehabilitation research, and the second consensus meeting will include their updates. Based on that discussion, the following eight topics of interest were identified for the RWGs in the Kobe meeting (Table 2): (a) patient selection (population); (b) blinding; (c) treatment group; $(d)$ control group and co-interventions; $(e)$ attrition, follow-up, and protocol deviation; $(f)$ outcomes; $(g)$ statistical analysis and appropriate randomization; and $(h)$ generalities on research (design, research question, effectiveness).

\section{The RCTRACK Working Groups}

The RWGs include participants recruited by the leaders nominated by the Executive Committee (Table 2).

The scope of the RWGs is to carry out studies to supplement the findings from the preliminary works toward development of RCTRACK ${ }^{1,4,9-18,27}$ and to identify the specific items for inclusion in the RCTRACK checklist. The procedure will include the following: analysis of all the items coming from the preliminary studies; checking of the articles referenced in the preliminary studies; and deciding on the relevant literature to be included. Electronic databases as well as manual searches will be done by each RWG. A synthesis of the literature (either narrative or quantitative, depending on the available evidence) will be performed and potential items/issues to be included/ added in the rehabilitation specific tools will be compiled. This information will be presented and discussed in the second consensus meeting by the leader of each RWG. After the consensus meeting, each RWG will prepare an article with the information compiled. Potential items, their correspondent definition, and examples for them to be used in the RCTRACK checklists will be also summarized in the articles. These articles will be reviewed by the Executive and Advisory Committees of RCTRACK and submitted to a major rehabilitation journal to form a special issue that will include all the preparation systematic/scoping reviews of the RCTRACK Project.

\section{Phase 3: Guidelines Development}

This phase will involve compiling information from the previous phases and generating a draft document with the guidelines through a second consensus meeting.

A 2-day consensus meeting will be held in Orlando during the 4th CRMM in March 2020. It will start with the reporting on two preliminary projects: the first results of the "rehabilitation definition" project from the consensus meeting the 3rd CRMM in Milan, February 13-14, 2020, and the update of the reporting and conduct checklists in physical therapy. ${ }^{27}$ In addition, previous experiences of relevant reporting guidelines will be presented. ${ }^{37-39}$ Then, RWG leaders will present the results of their systematic/scoping reviews. Everything will be thoroughly discussed by the Executive Committee to identify the set of specific items for the first draft of the RCTRACK checklists. The item(s) and relevant description(s) proposed to be included in the checklists will be discussed, corrected, and integrated. At the end of the meeting, the first draft version of RCTRACK checklists will be approved. 


\section{Phase 4: Delphi Process Consensus}

The draft version of the RCTRACK checklist will be submitted to a series of Delphi Rounds, as many as needed. The participants for the Delphi procedure will be recruited by Cochrane Rehabilitation and by the RCTRACK Executive and Advisory Board. They will consist of all the English-reading people agreeing to participate including the following: $(a)$ authors of articles on methodological issues in rehabilitation research, $(b)$ members of editorial boards of rehabilitation journals, $(c)$ members of groups dealing with evidence and methodology in rehabilitation, $(d)$ members of Cochrane methods groups, $(e)$ authors of Cochrane Reviews relevant to rehabilitation, $(f)$ authors of RCTs published in rehabilitation journals, $(g)$ members of the methodological group who developed checklists relevant to rehabilitation, and $(h)$ members of patients groups and organizations.

Each expert will be asked to rate the relevance of the items for evaluating reporting or conduct of RCTs in rehabilitation using a Likert scale. Recommendations on relevant items will be drafted by the Executive Committee and presented to the expert panel in two or three rounds (or more if necessary) of Internet-based surveys. Recommendation-specific medians will be estimated for each round. Items considered relevant by less than $10 \%$ and more than $90 \%$ of experts will be discarded and accepted, respectively. All other items will be deferred to the second round. Experts will be provided with both qualitative and quantitative feedback after each round. Experts will have the opportunity to add comments or provide free suggestions for discussion after each round. New versions of the checklists will be circulated until an agreement is obtained.

\section{Phase 5: Final Consensus Meeting and Paper Production}

The Executive Committee will meet for the last time at the end of the Delphi Consensus to discuss the final recommendations and resolve remaining issues through a formal voting process. At the end of this meeting, the final RCTRACK Guidelines will be made public.

The writing of the final report will be the responsibility of the project leadership. The RWGs leaders will be in charge of writing the final version of the definitions and explanations for the manual according to the decisions made in the Delphi Rounds. The Executive Committee will be in charge of revising and accepting the final version of the manuscript. The Advisory Committee will receive the final version for comments and review. The article will be published in a recognized medical journal and co-published in all rehabilitation journals that will accept and apply the guidelines. In addition, journals not publishing the article will be invited to implement the utilization of the RCTRACK guidelines.

\section{CONCLUSIONS}

We expect RCTRACK to be an important contribution to research and practice in the field of rehabilitation. This work will be valuable to a wide variety of stakeholders: researchers, systematic reviewers and meta-analysts, methodologists, clinicians, patients, guideline developers, and policy-makers working in this area. The RCTRACK will potentially impact reporting and conduct quality of future RCTs, systematic reviews, and clinical practice guidelines in rehabilitation. In addition, the use of a wide Delphi to develop recommendations into a consensus document will enhance dissemination.

The RCTRACK is in part a knowledge translation project, as expected from Cochrane Rehabilitation, ${ }^{7}$ because it will compile all the items relevant to rehabilitation previously published in other guidelines. ${ }^{29}$ Furthermore, the RCTRACK will be an original contribution because it will identify specific problems that are unique to rehabilitation ${ }^{4}$ to generate some new items. Finally, we expect RCTRACK recommendations to improve the understanding about rehabilitation among clinician and scientists in other fields, the Cochrane network, and editors of journals.

The RCTRACK Promoters include the following: Stefano Negrini (Ita), Chiara Arienti (Ita), Susan Armijo-Olivo (Ger/ Can), Julia Patrick Engkasan (Mys), Walter Frontera Roura (USA), Allen W. Heinemann (USA), Wendy Machalicek (USA), Frane Grubisic (Hrv), Carlotte Kiekens (Bel), William Levack (Nzl), Antti Malmivaara (Fin), Thorsten Meyer (Ger), Aydan Oral (Tur), Melissa Selb (Che), Gerold Stucki (Che), Will Taylor (Nzl), and John Whyte (USA).

\section{REFERENCES}

1. Negrini S, Arienti C, Pollet J, et al: Clinical replicability of rehabilitation interventions in randomized controlled trials reported in main journals is inadequate. $J$ Clin Epidemiol 2019;114:108-17

2. Negrini S, Levack W, Gimigliano F, et al: The struggle for evidence in physical and rehabilitation medicine: publication rate of randomized controlled trials and systematic reviews is growing more than in other therapeutic fields. Am J Phys Med Rehabil 2019;98:258-65

3. Levack WMM, Rathore FA, Pollet J, et al: One in 11 Cochrane Reviews are on rehabilitation interventions, according to pragmatic inclusion criteria developed by Cochrane Rehabilitation. Arch Phys Med Rehabil 2019;100:1492-8

4. Arienti C, Armijo-Olivo S, Minozzi S, et al: 60 methodological issues in rehabilitation research: a scoping review. BMJ Evid Based Med 2019;24(suppl 1):A35

5. Arienti C, Buraschi R, Pollet J, et al: Opening the black box of 'usual care' and finding a black hole: a numerical systematic review on 'usual care' control groups in stroke rehabilitation RCTs. BMJ Evid-Based Med 2019;24(suppl 1):A27-8

6. Negrini S, Arienti C, Gimigliano F, et al: Cochrane Rehabilitation: organization and functioning. Am J Phys Med Rehabil 2018;97:68-71

7. Negrini S, Gimigliano F, Arienti C, et al: Knowledge translation: the bridging function of Cochrane Rehabilitation. Arch Phys Med Rehabil 2018;99:1242-5

8. Levack WM, Meyer T, Negrini S, et al: Cochrane Rehabilitation Methodology Committee: an international survey of priorities for future work. Eur J Phys Rehabil Med 2017;53:814-7

9. Levack WM, Malmivaara A, Meyer T, et al: Methodological problems in rehabilitation research. Report from a cochrane rehabilitation methodology meeting. Eur J Phys Rehabil Med 2019;55:319-21

10. Stucki G, Pollock A, Engkasan JP, et al: How to use the International Classification of Functioning, Disability and Health as a reference system for comparative evaluation and standardized reporting of rehabilitation interventions. Eur J Phys Rehabil Med 2019;55:384-94

11. Malmivaara A: The human risks of bias in medical and rehabilitation research and practice: the eight is. Eur J Phys Rehabil Med 2019;55:372-7

12. Kayes NM, Martin RA, Bright FA, et al: Optimizing the real-world impact of rehabilitation reviews: increasing the relevance and usability of systematic reviews in rehabilitation. Eur J Phys Rehabil Med 2019;55:331-41

13. Levack WM, Martin RA, Graham FP, et al: Compared to what? An analysis of the management of control groups in Cochrane reviews in neurorehabilitation. Eur J Phys Rehabil Med 2019;55:353-63

14. Engkasan JP, Ahmad-Fauzi A, Sabirin S, et al: Mapping the primary outcomes reported in Cochrane systematic reviews regarding stroke with the International Classification of Functioning, Disability and Health domains: current trend and future recommendations. Eur J Phys Rehabil Med 2019;55:378-83

15. Hay-Smith EJ, Englas K, Dumoulin C, et al: The Consensus on Exercise Reporting Template (CERT) in a systematic review of exercise-based rehabilitation effectiveness: completeness of reporting, rater agreement, and utility. Eur J Phys Rehabil Med 2019;55:342-52

16. Taylor WJ, Green SE: Use of multi-attribute decision-making to inform prioritization of Cochrane review topics relevant to rehabilitation. Eur J Phys Rehabil Med 2019;55:322-30

17. Meyer T, Wulff K: Issues of comorbidity in clinical guidelines and systematic reviews from a rehabilitation perspective. Eur J Phys Rehabil Med 2019;55:364-71 
18. Pollock A, van Wijck F: Cochrane overviews: how can we optimize their impact on evidence-based rehabilitation? Eur J Phys Rehabil Med 2019;55:395-410

19. Negrini S, Meyer T, Arienti C, et al: Cochrane Rehabilitation Methodology Meeting participants: In search of solutions for evidence generation in rehabilitation: The second Cochrane Rehabilitation Methodology Meeting. Am J Phys Med Rehabil 2020;99:181-2

20. Malmivaara A, Armijo-Olivo S, Dennett L, et al: Blinded or nonblinded randomized controlled trials in rehabilitation research: A conceptual analysis based on a systematic review. Am J Phys Med Rehabil 2020;99:183-90

21. Armijo-Olivo S, Dennett L, Arienti C, et al: Blinding in rehabilitation research: Empirical evidence on the association between blinding and treatment effect estimates. Am J Phys Med Rehabil 2020;99:198-209

22. Meyer T, Selb M, Kiekens C, et al: Toward better reporting standards of patients' characteristics in rehabilitation trials: Applying a new conceptual framework to current standards. Am J Phys Med Rehabil 2020;99:216-23

23. Levack WMM, Engkasan JP, Heinemann AW, et al: A review of CONSORT guidelines about comparison groups with a focused discussion on implications for rehabilitation clinical trials. Am J Phys Med Rehabil 2020;99:191-7

24. Armijo-Olivo S, Fuentes J, da Costa BR, et al: Blinding in physical therapy trials and its association with treatment effects: a meta-epidemiological study. Am J Phys Med Rehabil 2017;96:34-44

25. Armijo-Olivo S, Saltaji H, da Costa BR, et al: What is the influence of randomisation sequence generation and allocation concealment on treatment effects of physical therapy trials? A meta-epidemiological study. BMJ Open 2015;5:e08562

26. Armijo-Olivo S, Stiles CR, Hagen NA, et al: Assessment of study quality for systematic reviews: a comparison of the Cochrane Collaboration Risk of Bias Tool and the Effective Public Health Practice Project Quality Assessment Tool: methodological research. J Eval Clin Pract 2012;18:12-8

27. Armijo-Olivo S, Fuentes J, Ospina M, et al: Inconsistency in the items included in tools used in general health research and physical therapy to evaluate the methodological quality of randomized controlled trials: a descriptive analysis. BMC Med Res Methodol 2013;13:116
28. Armijo-Olivo S, Macedo LG, Gadotti IC, et al: Scales to assess the quality of randomized controlled trials: a systematic review. Phys Ther 2008;88:156-75

29. Armijo-Olivo S, da Costa BR, Cummings GG, et al: PEDro or Cochrane to assess the quality of clinical trials? A meta-epidemiological study. PloS One 2015;10:e132634

30. Armijo-Olivo S, Cummings GG, Fuentes J, et al: Identifying items to assess methodological quality in physical therapy trials: a factor analysis. Phys Ther 2014;94:1272-84

31. Colle F, Rannou F, Revel M, et al: Impact of quality scales on levels of evidence inferred from a systematic review of exercise therapy and low back pain. Arch Phys Med Rehabil 2002;83:1745-52

32. Herbison P, Hay-Smith J, Gillespie WJ: Adjustment of meta-analyses on the basis of quality scores should be abandoned. J Clin Epidemiol 2006;59:1249-56

33. Jüni P, Witschi A, Bloch R, et al: The hazards of scoring the quality of clinical trials for meta-analysis. JAMA 1999;282:1054-60

34. Whyte J, Dijkers MP, Van Stan JH, et al: Specifying what we study and implement in rehabilitation: comments on the reporting of clinical research. Arch Phys Med Rehabil 2018;99:1433-5

35. Boutron I, Moher D, Altman DG, et al: CONSORT Group: Methods and processes of the CONSORT group: example of an extension for trials assessing nonpharmacologic treatments. Ann Intern Med 2008;148:W60-6

36. Boutron I, Moher D, Altman DG, et al: CONSORT Group: Extending the CONSORT statement to randomized trials of nonpharmacologic treatment: explanation and elaboration. Ann Intern Med 2008;148:295-309

37. Slade SC, Dionne CE, Underwood M, et al: Consensus on Exercise Reporting Template (CERT): modified Delphi study. Phys Ther 2016;96:1514-24

38. Groeneweg R, Rubinstein SM, Oostendorp RAB, et al: Guideline for Reporting Interventions on Spinal Manipulative Therapy: Consensus on Interventions Reporting Criteria list for Spinal Manipulative Therapy (CIRCLe SMT). J Manipulative Physiol Ther 2017;40:61-70

39. Hoffmann TC, Glasziou PP, Boutron I, et al: Better reporting of interventions: Template for Intervention Description and Replication (TIDieR) checklist and guide. $B M J$ 2014;348:g1687 